\title{
The influence of emerging low mood symptoms on sleep in children: a pilot study
}

\author{
This article was published in the following Dove Press journal: \\ Nature and Science of Sleep \\ 12 October 2012 \\ Number of times this article has been viewed
}

\section{Deirdre A Conroy \\ Anameti Usoro \\ Robert F Hoffmann \\ Kirk J Brower \\ Roseanne Armitage}

University of Michigan, Department of Psychiatry, Ann Arbor, MI, USA
Correspondence: Deirdre A Conroy 4250 Plymouth Road Ann Arbor,

MI, 48I09, USA

Tel +l 7342320559

Fax +I 7349987992

Email daconroy@umich.edu
Purpose: Sleep disturbances can lead to the onset and relapse of psychiatric disorders. However, the age at which this relationship begins and the role of sleep disturbances in the trajectory to the onset of a psychiatric disorder are still not fully understood. The purpose of this study was to explore, based on self- and parental-reports of mood symptoms, subjective and objective sleep in young children who are at risk of developing a psychiatric disorder but who have not yet met diagnostic criteria.

Patients and methods: Twenty-one children (eleven girls) between the ages of 8 and 11 (mean age $=9.7$ years, standard deviation $=1.1$ years) were dichotomized into low mood (LM) and not low mood (NLM) groups based on scoring below or above the median threshold score on at least two of the following questionnaires: the Child Depressive Rating Scale (CDRS), Weinberg Screening Affective Scale (WSAS), and Quick Inventory of Depressive Symptomatology (QIDS). The children completed sleep diaries and underwent two nights (for adaptation and baseline) of polysomnography. Sleep stages and sleep microarchitecture (alpha, sigma, beta, and delta) in the first half of the night, were analyzed.

Results: Self-reported sleep disturbance accounted for $72 \%$ of the variance $(\mathrm{F}[3,20]=15$, $P<0.005$ ) of the Weinberg Screening Affective Scale in LM children. LM children had fewer arousals at night, but awakened earlier than NLM children. Regardless of mood, girls had more sleep disturbance, as well as lower alpha, beta, and delta power in the first half of the night, compared to boys. Girls with LM had shorter sleep times and a lower percentage of rapid eye movement sleep.

Conclusions: Girls with and without LM, and without a clinical diagnosis of depression, showed more sleep disturbances than boys of the same age. Sleep disturbances evident early in life and in LM girls may reflect greater risk for future sleep or psychiatric disorders.

Keywords: depression, insomnia, EEG, pediatrics

\section{Introduction}

A number of studies have shown that insomnia can predispose an individual to developing a psychiatric disorder over time. A large prospective study on individuals without insomnia at baseline, revealed that, 7.5 years later, women had a higher incidence of insomnia than men, and that poor sleep and mental health were stronger predictors of insomnia than physical health. ${ }^{1}$ Epidemiological studies have shown that once insomnia develops, it can predict alcohol use, depression, anxiety, and substance abuse several years later. ${ }^{2-4}$ A meta-analysis showed that nondepressed individuals with insomnia were 2.6 times more likely to develop depression. ${ }^{5}$ These studies suggest there may be a developmental time course by which sleep disruption begins to be associated with other symptomatology and the eventual onset of disorders such 
as depression and alcohol dependence. However, the age at which this might occur and how sleep disturbances play a role in the trajectory to the onset of a psychiatric disorder are still not fully understood.

Much of our knowledge about the mechanisms of sleep disturbance in psychiatric disorders derives from studies conducted in adults several years after initial diagnosis. For example, subjective sleep complaints are common in up to $90 \%$ of both alcohol dependent ${ }^{6,7}$ and depressed patients. ${ }^{8}$ Objective findings in alterations of sleep stages (also called sleep macroarchitecture) in depression ${ }^{8}$ and alcohol dependence $^{6}$ support these findings.

Sophisticated analyses of sleep electroencephalography (EEG) (also called microarchitecture) across the night have now been able to differentiate alcohol-dependent (AD) adults from adults with major depressive disorder and healthy controls. These studies examine slow wave activity (SWA) in the first part of the night in response to a sleep delay (ie, staying up 3 hours past habitual bedtime) as a way to measure how individuals discharge the amount of sleep debt accumulated across the day. ${ }^{9}$ Men with major depressive disorder have been shown to generate less SWA in the first non-rapid eye movement (NREM) period in response to a sleep challenge, when compared to healthy controls and men with AD. ${ }^{10}$ SWA dissipation in AD men and women shows a similar pattern of blunted SWA response to sleep delay, with significantly lower SWA power in ADs than in healthy controls. ${ }^{11}$ Whether these EEG differences are predisposing risk factors (and therefore potential biological markers) or a consequence of the disorder is still unclear.

Recent studies have begun to measure sleep in highrisk children and adolescents who have a family history of a psychiatric illness, but have yet to be diagnosed with a psychiatric disorder. Polysomnography (PSG) studies have evaluated sleep in children and adolescents based on their having a parent with alcohol dependence, ${ }^{12,13}$ depression, ${ }^{14}$ or having already met criteria for depression. ${ }^{12,14,15}$ No differences in visually scored sleep measures have been found in children at high risk for alcoholism, whereas children at high risk for depression have shown less time in stage 1 sleep, fewer arousals during the night, less time in wakefulness in the night, and higher sleep efficiency. ${ }^{14}$ Last, Rao et $\mathrm{al}^{16}$ found that changes in REM sleep predicted later depression in adolescents (mean age 15) with a family history of a psychiatric disorder.

Subtle EEG findings in sleep microarchitecture (ie, power across a range of EEG frequency bands) have distinguished children with a family history of alcohol dependence (COAs) and with a family history of depression. COAs between 9 and 10 years old and without a personal or family history of other psychopathologies showed less normalized power in high spindle frequencies (defined as 13-16 Hz) and slower frequency EEG frequencies (defined as 3-6 Hz) during NREM sleep in the first half of the night. This finding was thought to indicate that decreased spindle activity may reflect a greater vulnerability to sleep disruption in this group. The low delta power was purported to reflect possible impairment in homeostatic mechanisms of sleep in COAs. ${ }^{13}$ Adolescents with a personal and family history of depression had decreased sleep spindle activity (defined as $11-16 \mathrm{~Hz}$ ), particularly girls; ${ }^{14}$ increased power in the slow alpha frequency band $(7.5-11 \mathrm{~Hz})$ in both the first and second NREM and REM sleep periods; and in boys, increased delta frequencies $(0.75-4.50 \mathrm{~Hz})$ during the second REM period. ${ }^{12}$ In summary, despite modest group differences in sleep macroarchitecture in high-risk children, some sleep EEG measures do distinguish these children from healthy controls.

No study has evaluated sleep in children with prodromal symptoms of a psychiatric disorder. Studies conducted to date have evaluated either high-risk children based on family history or high risk based on already meeting diagnostic criteria. Moreover, many of the participants in previous studies ${ }^{12,14}$ are older than age 14, which is generally after the hormonal changes of puberty have begun and is the mean age at which drinking alcohol tends to start. Therefore, it is difficult to account for sleep outside of the influences of hormones and drinking, and perhaps drug use as well. Finally, while PSG measures are the gold standard of sleep measures, they may not be the best for fully characterizing sleep disturbance. Using subjective rating scales, high-risk teenage girls with maternal history of depression ${ }^{17}$ described their sleep as being of poorer quality than did low-risk girls, but neither groups' diaries or actigraphy described differences in sleep. Therefore, both subjective and objective assessment of sleep in this sample are critical.

The current study focuses on young children who do not meet criteria for a psychiatric diagnosis, but are beginning to report subclinical signs of mood problems. We are also studying prepubertal children because sleep undergoes such rapid changes during adolescence ${ }^{18}$ (linked to puberty and to social and environmental influences). Our goal was to identify any potential differences in sleep between two groups, that is, below and above a set threshold for mood problems. We were interested in prodromal symptoms of a mood disorder, and this threshold does not reflect a clinical DSM-IV diagnosis of depression. Based on our previous work, ${ }^{14,15,19}$ we propose that sleep architecture may begin to change early in childhood 
development and represent a marker of elevated risk for the development of a psychiatric disorder ${ }^{19}$ (eg, depression or substance use disorder). We hypothesized that (1) children who are beginning to show signs of a mood disorder will (a) report more sleep disturbances subjectively, and (b) show greater sleep disruption via PSG; and (2) these findings would be more pronounced in girls.

\section{Materials and methods \\ Participants}

Twenty-one children (eleven girls) between the ages of 8 and $11($ mean $=9.7$ years, standard deviation $=1.1)$ were recruited from flyer postings around the larger University of Michigan Health System, the Depression Center in the Department of Psychiatry, Internet postings on Craigslist. org, and other studies involving individuals with alcohol dependence. One child (female) completed all paper questionnaires, but chose not to undergo sleep study testing; therefore, the sleep study shows data for ten boys and ten girls. This study was approved by the University of Michigan Institutional Review Board.

\section{Screening}

Parents completed phone screens about their children's sleeping patterns to exclude children with known sleeping disorders. Each child was later assessed for psychiatric and mood disorders using the Schedule for Affective Disorders and Schizophrenia for School-Age Children - Present and Lifetime Version ${ }^{20}$ during an interview with the child. Upon arrival at the sleep laboratory, the parent and child underwent an evaluation by a clinical psychologist. Parents completed a demographic questionnaire and a general medical history questionnaire about the child. The parent(s) were asked about the family history of psychiatry disorders (eg, alcoholism and depression) in the child's first-,second-, and third-degree relatives. Children with medical or mental illnesses were excluded. All children in the study were healthy: children with no medical or mental illnesses - acute or chronic. No child was taking medications of any kind.

\section{Mood instruments}

\section{Quick inventory of depressive symptomatology}

The parent completed the Quick Inventory of Depressive Symptomatology (QIDS) ${ }^{21}$ on behalf of the child in the evening of the adaptation night in the sleep laboratory. The QIDS is a 16-item test that assesses for nine criteria domains for depression: sleep disturbance, sadness, appetite changes, weight changes, concentration/decision making, view of oneself, thoughts of death or suicide, interests, and changes in energy level.

\section{Children's Depression Rating Scale}

The Children's Depression Rating Scale (CDRS) ${ }^{22}$ was administered on the day of the screening interview. The CDRS is a brief rating scale designed for children 6-12 years of age that is based on a semistructured interview with the child. The interviewer rates 17 symptom areas on a 7-point scale and generates a $t$-score.

\section{Weinberg Screening Affective Scale}

The child completed the Weinberg Screening Affective Scale (WSAS) ${ }^{23}$ in the evening of the adaptation night in the sleep laboratory. The WSAS has 56 yes/no questions that are completed by the child. Items are classified into ten categories: (1) dysphoric mood, (2) self-deprecatory ideation, (3) agitation, (4) sleep disturbance, (5) change in school performance, (6) diminished socialization, (7) change in attitudes toward school, (8) somatic complaints, (9) loss of usual energy, and (10) usual change in appetite.

\section{Family environment questionnaires}

\section{Family Global Assessment Scale}

With the Family Global Assessment Scale (FGAS), ${ }^{25}$ the parent(s) were asked to rate the family's functioning over the past year by considering the following areas: (1) social functioning of parents as related to economic and social goals, (2) marital/parental teamwork, (3) parental understanding and provision for the developmental needs of the child, and (4) integrity and stability of the family relationships. A rating of 1-100 was provided. For example, 1-10 indicated prolonged or permanent disruption of family functioning and 91-100 indicated superior functioning in all areas.

\section{Family Environment Scale}

The Family Environment Scale (FES) ${ }^{26}$ is a 90 -item scale with true/false responses completed by the parent. Of the 90 items, ten subscales are generated that include family cohesion, expressiveness, conflict, independence, achievementorientation, intellectual-cultural orientation, active-recreational orientation, moral-religious emphasis, organization, and family control. Summary scores were then summed.

\section{Sleep instruments \\ Sleep diaries}

The children completed a week-long sleep diary, which was verified by actigraphy. Children slept on their own habitual 
sleep/wake schedule. In the sleep diary, the children recorded the following items for each night: time lights were turned out, how long it took to fall asleep, how long they were awake during the night, final wake time, if they took naps during the day, and the duration of the naps. They rated the quality of their sleep on a scale of 1 to $3: 1=$ poor, $2=$ fair, $3=$ good.

\section{Polysomnography}

After the seven days of completing sleep diaries, the child slept in the laboratory for two consecutive nights. On the first night of study, a full clinical polysomnogram assessment was conducted to verify the absence of sleep disorders. Nasal oral thermistors were included for monitoring airflow, a chest band assessed respiratory effort, and tibialis electrodes were included for assessing periodic limb movements and restless legs. Oximetry was also included to assess oxygen desaturation, and EKG electrodes were included to assess electrocardiography. The PSG data from the screening night was then read by a board-certified sleep specialist (D Conroy) to ensure that no participant had an independent intrinsic sleep disorder. After screening, these breathing-monitoring and leg leads were discontinued, as is standard procedure. Data from the second night were used in this study. EEG was recorded from left and right frontal central, occipital, and parietal electrode sites according to the International 10-20 System. ${ }^{27}$ Electromyography was recorded from a bipolar chin-cheek montage. Monopolar and bipolar electro-oculography recordings were made from the supraorbital and infraorbital ridges of the eyes. The monopolar electro-oculography recordings facilitate sleep stage scoring, reflecting binocularly synchronous eye movements in REM. The bipolar montage is used by the computer algorithm to measure electro-oculography count and amplitude. Primary outcome variables from PSG include: total sleep time, sleep onset latency, stage 1 (N1), stage 2 (N2), stage 3 and 4 (N3) percentages, REM sleep percentage, REM sleep latency, number of arousals across the sleep period, percentage time spent awake and moving, the time spent awake before the scheduled wake time (early awakening), and sleep efficiency (\%).

\section{Power spectral analysis}

During visual scoring, epochs were tagged for artifact rejection. Epochs with any movement or electrical artifact, baseline shift, or electrode problems were excluded from analysis. Power spectral analysis was performed on digitized EEG signals. Delta power was defined as $0.5-3.9 \mathrm{~Hz}$, alpha as 8.0-11.9 Hz, sigma as $12.0-15.9 \mathrm{~Hz}$, and beta as $16-32 \mathrm{~Hz}$. The power spectral analysis algorithm, based on a fast Fourier transform, was taken from Press et al, ${ }^{26}$ and processed data in 2-second epochs (512 samples for each 2 seconds) with a Hanning window taper is the correct term. Hanning window taper. The power spectral analysis generated power (area under the curve) in the respective band expressed as $\mu V 2$. Power was then averaged in 30-second epochs to provide epoch lengths identical to the stage-score data. The definition of NREM periods closely followed that outlined by Dijk et $\mathrm{al}^{9,28-33}$ and Feinberg. ${ }^{34}$ NREM periods were defined as the succession of stages 2, 3, or 4 of 15 minutes in duration and terminated by REM sleep or a period of wakefulness of at least 5 minutes. Stage 1 sleep epochs were excluded from delta power. Because we anticipated many of the power spectral analyses to differ in the first half of the night, based on previous studies in both children ${ }^{12,13}$ and adults, ${ }^{10,11}$ power spectral analyses were done for the first half of the night (ie, first and second NREM and REM periods), as well as for the entire night.

\section{Data analysis}

Independent samples $t$-tests and chi-square tests were conducted to compare diagnostic groups on demographic and questionnaire data. Pearson correlational analyses were conducted to assess statistical relationships between variables. Multiple regression analyses analyzed the relationship between mood and sleep variables. All statistical analyses were two-tailed, they used a $P$-value of $<0.05$ for significance, and they were performed using the Statistical Package for Social Sciences (SPSS), version 17.0 (IBM Corporation, Chicago, IL). Values in the text are presented as means and standard deviation.

\section{Results}

\section{Demographic and clinical characteristics}

The children were dichotomized into low-mood or not-lowmood groups based on scoring below or above the median threshold score on at least two of the following three questionnaires: the QIDS, CDRS, and WSAS. The median score of the QIDS was 3, the median CDRS T-score was 33, and the median number of positive categories on the WSAS was three. Of the total sample, three $(14.3 \%)$ did not meet any criteria for any questionnaire, six $(28.6 \%)$ met criteria on one questionnaire, eight $(38.1 \%)$ met criteria on two questionnaires, and four $(19.0 \%)$ met criteria on all three questionnaires. Of the twelve children classified as having low mood (LM), eight (66.7\%) met criteria on two questionnaires, and four $(33.3 \%)$ met criteria on three questionnaires. Six (50\%) were girls, eight $(66.7 \%)$ had a parent with alcoholism, and five $(41.7 \%)$ had a parent with depression. Of the nine not 
low mood (NLM) children, three (33.3\%) did not meet any criteria on the questionnaires, and six (66.7\%) met criteria on one questionnaire (two on QIDS, three on CDRS, and one on WSAS). Five (55.6\%) were girls, three (33.3\%) had a parent with alcoholism, and none had a parent with depression. Table 1 describes other participant characteristics.

\section{Sleep characteristics}

Independent $t$-tests by mood status and by family history of depression did not reveal any significant differences in sleep-diary reports. An overall, significant difference as a function of mood, sex, or their interaction was observed in six of twelve PSG variables (Table 2). When the sample was split by children with a parent with alcohol dependence $(n=11)$, COAs reported better sleep quality $(2.8 \pm 0.4)$ than non-COAs $(\mathrm{n}=10 ; 2.4 \pm 0.4, \mathrm{t}=-2.4$, $P=0.03$ ). When controlling for age, a sex-by-mood analysis of variance (ANOVA) did not reveal any significant main effects or interaction effects for any of the sleep-diary variables.

The impact of mood and sex on sleep macroarchitecture is displayed in Table 2. There was a trend toward a main effect of mood $(P=0.05)$ and $\operatorname{sex}(P=0.03)$ on the number of arousals across the night, such that LM girls $(n=5)$ had a lower number of arousals in the total sleep period than NLM girls $(\mathrm{n}=5)(18 \pm 7.3$ versus $30 \pm 7.0, \mathrm{t}=3.3, P=0.03)$. There was no difference in number of arousals between LM boys and NLM boys. Girls had a higher percentage of stage 1 sleep and a greater time spent awake during the night, compared to boys. NLM boys had the shortest total sleep time and girls with LM slept less than boys with LM (544 minutes \pm 17 vs $562 \pm 15$, Cohen's d $=-1.1$ ). A significant interaction between mood and sex was observed for REM\%, such that LM girls had a significantly lower REM sleep percentage than LM boys $(17 \% \pm 2.1 \%$ vs $22 \% \pm 3.5 \%$,
Cohen's d=-1.7). When collapsed across sex and controlling for age, children with LM $(n=11)$ spent a longer time awake in the morning than NLM children $(n=9)(18$ minutes \pm 18.5 versus 5 minutes \pm 8.1 ), and they had fewer arousals across the night $(17 \pm 5.9$ versus $25 \pm 8.9)$.

The impact of mood and sex on sleep microarchitecture is displayed in Table 3. There was no main effect of mood or interaction between sex and mood on any of the microarchitecture variables. Regardless of mood, girls had lower alpha, beta, and delta than boys.

\section{Family history of psychopathology}

A sex by family history (FH) of depression ANOVA was conducted in a small number of children with an $\mathrm{FH}$ of depression (FH-positive $n=5$ vs FH-negative $n=15$ ) on microarchitecture across the night. There was an interaction between sex and FH of depression on all bandwidths measured across the night. FH-positive girls had more beta across the night than FH-negative girls $(32 \pm 3.3 \mathrm{~Hz}$ vs $24.6 \pm 1.9 \mathrm{~Hz}$, $\mathrm{t}=0.27, P=0.005)$, and FH-positive boys had less beta power than FH-negative boys $(24 \pm 2.8 \mathrm{~Hz}$ vs $29 \pm 2.4 \mathrm{~Hz}, \mathrm{t}=0.96$, $P=0.023)$. FH-positive boys had lower delta power across the night than FH-negative boys $(628.6 \pm 24.2$ vs $747.7 \pm 66.4 \mathrm{~Hz}$, $\mathrm{t}=2.9, P=0.02)$. There was no difference in delta power between FH-positive and FH-negative girls. FH-negative boys had more alpha $(129.0 \pm 14.3)$ than FH-negative girls $(102.9 \pm 16.6, \mathrm{t}=-3.2, P=0.01)$ across the night.

When examining microarchitecture in the first half of the night, there was an interaction between $\mathrm{FH}$ of depression and sex on alpha in NREM1 $(\mathrm{F}[15,20]=5.6, P=0.03)$. There was no interaction between sex and family history of alcoholism on any of the bandwidths across the entire night, or in the first two NREM and REM periods.

Table I Demographic and clinical variables

\begin{tabular}{|c|c|c|c|c|c|c|c|}
\hline & \multicolumn{2}{|c|}{ Boys $(n=10)$} & \multicolumn{2}{|c|}{ Girls (n = I I) } & \multicolumn{3}{|c|}{ Statistical effects (F[I6, I9]) } \\
\hline & $\operatorname{LM}(n=5)$ & $\operatorname{NLM}(n=4)$ & $\operatorname{LM}(n=6)$ & $\operatorname{NLM}(n=5)$ & Mood & Sex & $\begin{array}{l}\text { Mood * } \\
\text { sex }^{\mathrm{a}}\end{array}$ \\
\hline Age (years) & $6.7(1.0)$ & $8.8(1.0)$ & $10.0(1.0)$ & $10.2(\mathrm{I} .3)$ & 0.59 & 3.7 & 1.4 \\
\hline Body mass index & $17.5(3.2)$ & $18.1(1.0)$ & $19.7(3.3)$ & $17.6(3.4)$ & 0.26 & 0.32 & 0.95 \\
\hline Tanner stage & $\mathrm{I} .0(0)$ & $\mathrm{I} .0(0)$ & $2.0(1.3)$ & $2.6(1.1)$ & 0.59 & II.I* & 0.59 \\
\hline$\%$ Caucasian & $4(67 \%)$ & $4(100 \%)$ & $6(100 \%)$ & $3(60 \%)$ & 0.18 & 0.18 & 20.9 \\
\hline FGAS & $69.7(11.8)$ & $79.5(9.8)$ & $82.3(7.7)$ & $80.6(17.1)$ & 0.51 & 1.8 & 1.3 \\
\hline FES & $52.8(5.8)$ & $57.8(10.7)$ & $58.8(7.0)$ & $62.0(6.1)$ & 1.6 & 2.5 & 0.08 \\
\hline WSAS & $3.5(2.9)$ & I.8(2.9) & $5.2(2.1)$ & $0.4(0.9)$ & $10.0 *$ & 0.02 & 2.1 \\
\hline CDRS (T-score) & $35.5(5.0)$ & $35.0(5.8)$ & $34.5(8.2)$ & $35.0(5.8)$ & 0.30 & 0.54 & 0.13 \\
\hline QIDS & $4.3(1.2)$ & I.3 (0.50) & $4.5(2.3)$ & $2.6(1.3)$ & $12.6 *$ & 1.2 & 0.71 \\
\hline
\end{tabular}

Notes: Variables with a significant main effect or interaction are in bold. Tanner stage is an index of pubertal development; WSAS scoring: number of low mood categories;

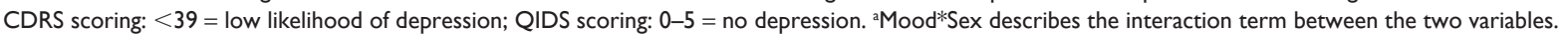

Abbreviations: FGAS, Family Global Assessment Scale; FES, Family Environment Scale; WSAS, Weinberg Screening Affective Scale; CDRS, Children's Depression Rating Scale; QIDS, Quick Inventory of Depressive Symptomatology. 
Table 2 Polysomnographic variables, mood, and sex

\begin{tabular}{|c|c|c|c|c|c|c|c|}
\hline & \multicolumn{2}{|c|}{ Boys $(n=10)$} & \multicolumn{2}{|c|}{ Girls $(n=10)$} & \multicolumn{3}{|c|}{ Statistical effects (F[16, 19]) } \\
\hline & $\operatorname{LM}(n=6)$ & $\operatorname{NLM}(n=4)$ & $\operatorname{LM}(n=5)$ & $\operatorname{NLM}(n=5)$ & Mood & Sex & $\begin{array}{l}\text { Mood * } \\
\text { sex }\end{array}$ \\
\hline Total sleep time (minutes) & $562(30)$ & $512(8.7)$ & $544(33)$ & $580(56)$ & 0.24 & 2.2 & $6.3^{\mathrm{b}}$ \\
\hline Sleep onset latency (minutes) & $37(30)$ & II (I3) & $14(5.0)$ & $18(15)$ & 1.0 & 1.0 & 2.3 \\
\hline Stage I\% (NI) & $0.65(0.15)$ & $0.79(\mathrm{I} . \mathrm{I})$ & $3.3(1.7)$ & $1.6(0.58)$ & 2.9 & $10.4^{b}$ & 3.7 \\
\hline Stage $2 \%(\mathrm{~N} 2)$ & $39(2.5)$ & $38(8.1)$ & $44(11.3)$ & $42(9.8)$ & 0.10 & 0.93 & 0.00 \\
\hline Stages $3 \%$ and $4 \%(\mathrm{~N} 3)$ & $36(5.6)$ & $39(9.6)$ & $30(8.5)$ & $28(8.5)$ & 0.01 & 2.6 & 0.23 \\
\hline REM\% & $22(3.5)$ & $20(2.2)$ & $17(2.1)$ & $21(3.5)$ & 1.2 & 4.2 & $4.7^{\mathrm{b}}$ \\
\hline REM latency & $124(6 \mid)$ & II 5 (42) & $137(56)$ & $98(30.7)$ & 1.4 & 0.07 & 0.18 \\
\hline Awake and moving \% & $2.0(0.4)$ & $2.7(1.9)$ & $5.5(3.2)$ & $6.5(3.9)$ & 0.63 & $5.7^{\mathrm{b}}$ & 0.00 \\
\hline Number arousals in TSP & $17(5.2)$ & $19(7.3)$ & $18(7.4)$ & $30(7.0)$ & 4.4 & $5.7^{\mathrm{b}}$ & 4.0 \\
\hline Early awakening (minutes) & $15(15.6)$ & $10(10.7)$ & $22(23)$ & $0.8(0.4)$ & $4.7^{\mathrm{b}}$ & 0.21 & 0.78 \\
\hline Sleep efficiency $\%$ & $90(5.2)$ & $93(3.5)$ & $89(3.6)$ & $90(4.8)$ & 1.4 & 0.62 & 0.15 \\
\hline
\end{tabular}

Notes: Variables with a significant main effect or interaction are in bold. ${ }^{\mathrm{a}}$ Controlling for age; ${ }^{\mathrm{b}} \leq 0.05$; Mood*Sex describes the interaction term between the two variables. Abbreviations: LM, Low mood; NLM, Not low mood; REM, Rapid eye movement sleep; TSP, Total sleep period.

\section{Relationship between mood and sleep}

Multiple regression analysis was used to test if LM, sex, or sleep disturbance significantly predicted total WSAS score. The results of the regression indicated that the three predictors explained $72 \%$ of the variance in the WSAS score $(\mathrm{R} 2=0.72, \mathrm{~F}[3,20]=15, P<0.005)$. Neither sex (beta $=-15, P=0.27$ ) nor LM (beta $=0.22, P=0.16$ ) independently predicted total WSAS score, but sleep disturbance $\operatorname{did}($ beta $=0.71, P<0.01$ ) (see Figure 1). A linear regression analysis also revealed that in girls, the total number of positive categories on the WSAS predicted sleep efficiency via sleep diary (beta $=0.67, P<0.05$ ), but not in boys (beta $=-0.31$, $P=0.39$ ).

Bivariate correlation revealed sleep onset latency captured via the sleep diary was correlated with objective sleep onset latency in LM children $(\mathrm{r}=0.84, P<0.005)$, but not in those without $\mathrm{LM}(\mathrm{r}=0.60, P=0.09)$. When conducting a partial correlation, controlling for family history of depression or alcoholism, this correlation remained significant, suggesting that family history did not have an influence on this relationship. There were no other significant correlations between subjective and objective sleep measures.

Table 3 Spectral power in frequency bands, mood, and sex in the first half of the night ${ }^{\mathrm{a}}$

\begin{tabular}{|c|c|c|c|c|c|c|c|}
\hline \multirow[t]{2}{*}{ Band } & \multicolumn{2}{|l|}{ Boys $(n=10)$} & \multicolumn{2}{|l|}{ Girls $(n=10)$} & \multicolumn{3}{|c|}{ Statistical effects (F[16, I9]) } \\
\hline & $\operatorname{LM}(n=6)$ & $\operatorname{NLM}(n=4)$ & $\operatorname{LM}(n=5)$ & $\operatorname{NLM}(n=5)$ & Mood (M) & $\operatorname{Sex}(\mathbf{S})$ & Mood* sex \\
\hline Alpha NREMI & $161.3(32.5)$ & $160.7(17.3)$ & $125.8(9.8)$ & $121.4(24.1)$ & 0.033 & $17.3^{b}$ & 0.37 \\
\hline Alpha REMI & $84.2(10.6)$ & $86.4(I I .0)$ & $72.4(8.2)$ & $87.6(25.9)$ & 1.3 & 0.37 & 0.81 \\
\hline Alpha NREM2 & $130.4(19.6)$ & $136.5(11.9)$ & $109.9(13.4)$ & $105.3(27.2)$ & 0.17 & $11.0^{\mathrm{b}}$ & 0.86 \\
\hline Alpha REM2 & $80.3(13.5)$ & $92.9(18.1)$ & $73.1(6.1)$ & $82.1(19.5)$ & 3.6 & 3.2 & 0.32 \\
\hline Alpha all night & $121.8(21.7)$ & I24.7 (I0.1) & I07.I (12.2) & I03.7 (20.8) & 0.06 & $6.4^{\mathrm{b}}$ & 0.43 \\
\hline Sigma NREMI & $78.3(18.4)$ & $76.0(4.5)$ & $71.8(11.9)$ & $64.6(12.6)$ & 0.25 & 3.5 & 0.40 \\
\hline Sigma REMI & $44.6(7.7)$ & $45.8(6.8)$ & $39.7(4.7)$ & $43.6(11.5)$ & 0.50 & 0.94 & 0.08 \\
\hline Sigma NREM2 & $66.3(15.7)$ & $65.3(5.1)$ & $66.1(12.1)$ & $61.8(16.1)$ & 0.04 & 0.54 & 0.24 \\
\hline Sigma REM2 & $4 I .0(8.4)$ & $50.6(10.0)$ & $39.5(5.1)$ & $40.8(8.3)$ & 2.7 & 2.7 & 1.7 \\
\hline Sigma all night & $64.0(15.3)$ & $63.3(3.4)$ & $63.9(10.4)$ & $63.3(3.4)$ & 0.10 & 0.68 & 0.40 \\
\hline Beta NREMI & $31.7(3.6)$ & $30.8(0.96)$ & $28.3(2.9)$ & $27.5(2.2)$ & 0.33 & $6.3^{b}$ & 0.00 \\
\hline Beta REMI & $21.4(3.1)$ & $23.4(3.1)$ & $22.7(4.7)$ & $24.4(5.2)$ & 0.66 & 0.59 & 0.00 \\
\hline Beta NREM2 & $26.3(2.4)$ & $27.4(1.7)$ & $23.9(2.6)$ & $25.5(2.9)$ & 2.2 & $5.0^{\mathrm{b}}$ & 0.00 \\
\hline Beta REM2 & 20.8 (2.9) & $24.8(3.3)$ & $20.6(2.2)$ & $21.4(2.3)$ & 3.3 & $\mathrm{I} .4$ & 1.3 \\
\hline Beta all night & $27.2(4.1)$ & $28.5(1.7)$ & $27.5(4.6)$ & $26.2(1.9)$ & 0.00 & 0.38 & 0.61 \\
\hline Delta NREMI & I I $94.9(233.7)$ & I I 30.7 (73.3) & 969.1 (129.4) & $939.6(142.8)$ & 0.28 & $6.3^{b}$ & 0.03 \\
\hline Delta REMI & $443.8(46.8)$ & $509.7(96.4)$ & 395.7 (82.8) & 484.1 (187.6) & 1.3 & 0.05 & 0.47 \\
\hline Delta NREM2 & $738.9(290.0)$ & $885.5(127.8)$ & $609.5(147.0)$ & $615.8(189.1)$ & 0.70 & 4.2 & 0.66 \\
\hline Delta REM2 & $4 \mid 9.2(58.4)$ & $518.2(85.6)$ & $374.6(92.0)$ & 405.5 (92.7) & 2.3 & 2.5 & 0.54 \\
\hline Delta all night & $687.9(94.6)$ & $748.1(37.0)$ & $606.8(67.7)$ & $597.2(91.9)$ & 0.32 & $6.7^{\mathrm{b}}$ & 0.68 \\
\hline
\end{tabular}

Notes: ${ }^{a}$ Controlling for age; ${ }^{b} \leq 0.05$; All EEG measures expressed in power $\left(\mathrm{mV}^{2}\right)$; Delta power $=0.5-3.9 \mathrm{~Hz}$, theta $=4.0-7.9 \mathrm{~Hz}$, alpha $=8.0-\mathrm{II} .9 \mathrm{~Hz}$, sigma $=12.0-15.9 \mathrm{~Hz}$, and beta $=16-32 \mathrm{~Hz}$. Variables with a significant main effect or interaction are in bold. Mood*Sex describes the interaction term between the two variables. Abbreviations: LM, Low mood; NLM, Not low mood; REM, Rapid eye movement sleep. 


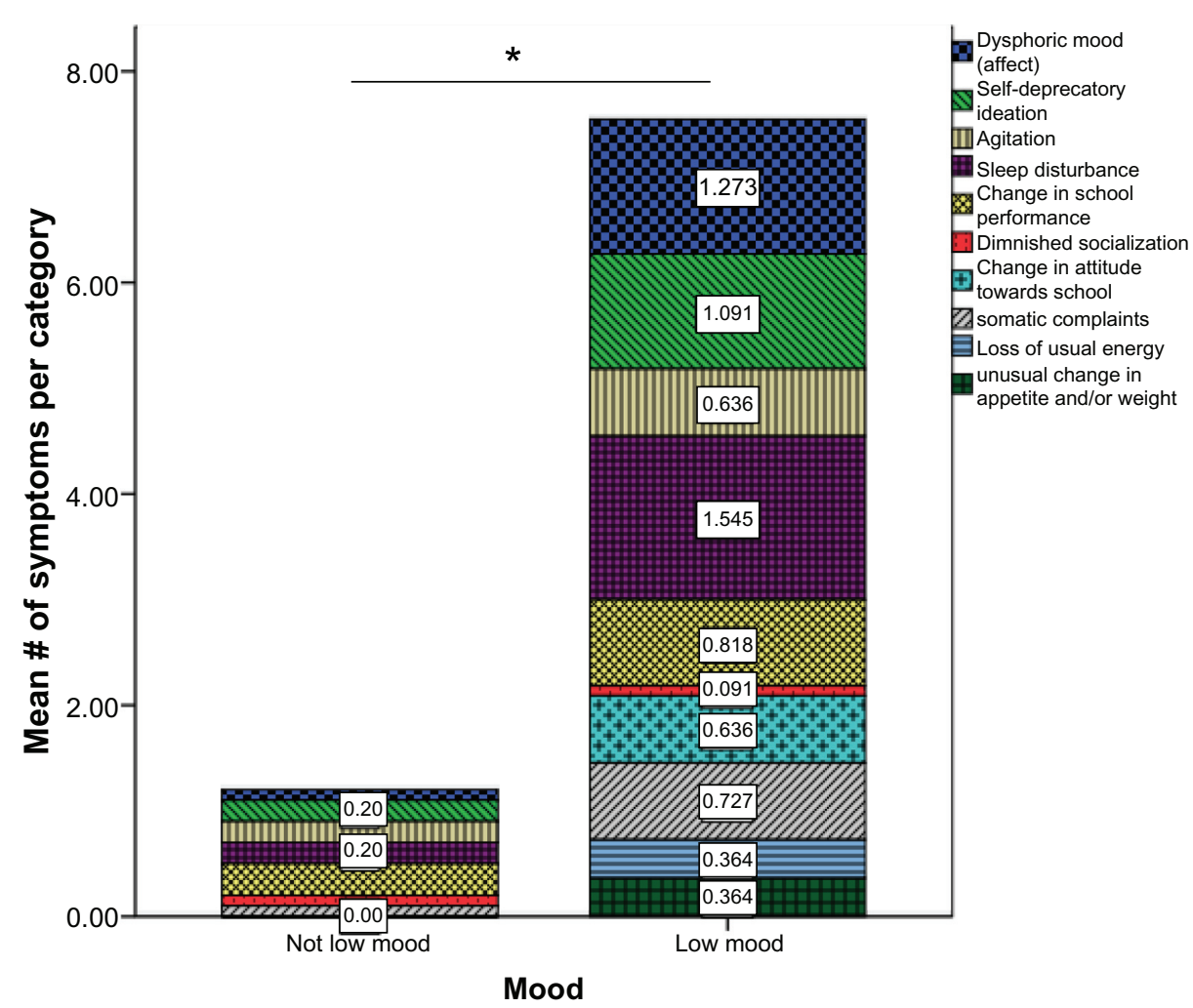

Figure I Self-reported mood symptoms on the Weinberg Screening Affective Scale between not low mood and low mood. *Indicates significant difference in mean number of symptoms between groups.

\section{Discussion}

The purpose of this study was to explore subjective and objective sleep in young boys and girls who are at risk for developing a psychiatric disorder based on emerging mood symptoms. We hypothesized (1) that children who are beginning to show signs of a mood disorder (a) would report more sleep disturbances subjectively and (b) would show greater sleep disruption via polysomnography, and (2) that these findings would be more pronounced in girls.

Our findings only partially supported our first hypothesis. Daily sleep diaries prior to the study revealed no differences on sleep measures between LM and NLM children. However, in the context of a mood questionnaire completed by the child (and not by the parent, such as on the other mood questionnaires), sleep disturbances accounted for a significant portion of the total score endorsed in LM children. The fact that LM children reported sleep disturbance is consistent with previous studies showing that insomnia is a common complaint in depressed children. ${ }^{35,36}$ Sleep-diary estimates of sleep onset latency in LM children were more consistent with the objective sleep latency measured by PSG, consistent with previous reports in a similar sample. ${ }^{35}$ Family history of depression was not associated with any pattern of subjective sleep assessments unlike another study, ${ }^{17}$ however, family history of alcoholism was associated with more positive assessment of sleep quality. The etiology of this finding is unclear.

Polysomnography showed the LM children were beginning to show sleep characteristics similar to those found in both adolescent depressed patients. LM children had longer early morning awakenings, a common complaint in depression and one that has been linked to multiple negative behavioral outcomes in adolescents. ${ }^{37} \mathrm{LM}$ children had fewer arousals during the night than NLM children, a finding consistent with Bertocci et a ${ }^{35}$ and Lopez et al. ${ }^{14}$ It is unlikely that this finding could be explained by prior partial sleep deprivation in this study, because all participants maintained regular sleep schedules (verified by actigraphy before the sleep laboratory study) and there were no differences in sleep reported in the diaries or in PSG on the adaptation night. The etiology of this consistent finding remains unclear.

Our third hypothesis was that sleep disturbances would be more pronounced in girls. Regardless of mood status, girls showed more sleep disturbances both in macro- and micro-architecture, compared to boys. Girls had less alpha power $(8.0-11.9 \mathrm{~Hz})$ than boys in the first two NREM periods, as well as across the whole night. Dahl et al ${ }^{12}$ also found a main effect of sex on both low $(7.50-11 \mathrm{~Hz})$ and 
high alpha amplitude (11.0-12.25 Hz) across the night in young depressed boys and girls. It is known that females are twice as likely to develop insomnia as males ${ }^{38}$ and that much of this may be due to hormonal influences. ${ }^{39}$ However, girls in this sample were all prepubertal, and analyses were statistically controlled for age; therefore, differences cannot be assumed to reflect hormonal or age differences. While the sample size in this study was quite small, these findings suggest that the higher risk of insomnia for girls may begin at an earlier age than previously thought, and as some studies now support, ${ }^{14,40}$ that this does not necessarily reflect the presence of a clinical disorder.

In the small number of boys and girls with a family history of depression in this study, there were interactions between sex and family history of depression on all bandwidths. Girls with a family history of depression had significantly more beta activity (16-32 Hz) in sleep compared to girls with no family history. Although the sample size was small, beta has been documented in adults with insomnia; ${ }^{41}$ this raises a question about the meaningfulness of this finding in individuals with a family history of depression that may reflect a predisposing factor to insomnia. We did not find a relationship in the family history-positive group with sigma (11-16 Hz), in contrast to Lopez et al. ${ }^{14}$ These findings may reflect our low statistical power or the fact that our sample was younger. Decreases in sleep spindles in high-risk children may decline with age.

This study also found that young girls with LM have less delta activity in the first two NREM periods. This is consistent with previous studies showing abnormal distribution of delta activity in non-REM sleep in adults with depression ${ }^{42}$ and may reflect impairment of homeostatic sleep drive. Given that there are no differences in sleep homeostasis from childhood to adolescence in healthy individuals, ${ }^{43}$ impairment in the ability to generate and discharge sleep "pressure" as measured by delta activity may be a biomarker of clinically significant mood changes or other psychiatric disorders in the future. This also suggests that the relationship between insomnia and psychiatric disorders may begin with a weakening of sleep/wake homeostasis that is evident at a young age.

Predisposition to poor sleep may have relevance to clinicians considering pharmacological treatment options. Studies have now shown that adolescents with depression and with insomnia are less likely to respond to serotoninselective reuptake-inhibitor medication regimens. ${ }^{44,45}$ Moreover, treatment-resistant depressed adolescents treated with such a regimen, plus an adjunctive sleep aid such as trazodone, were six times less likely to respond than those with no sleep medication. ${ }^{45}$ Early intervention strategies such as teaching young children, particularly girls, the importance of keeping a consistent sleep/wake schedule cannot be underestimated. Nonpharmacological insomnia treatment options for depressed youth and adolescents are needed.

There are limitations to the present study that warrant consideration. First, the sample size was small, which limited the statistical power of our comparisons, particularly interaction effects. Future research should include longitudinal studies of sleep in children with LM to help clarify the relationship between LM and later depression. Second, the sleep studies began at the children's typical bedtime. The most effective way to assess homeostatic regulation of delta activity would have been to conduct a sleep challenge ${ }^{10,11}$ (eg, subjects stay awake for later than their typical bedtime). Future studies should include a sleep-challenge protocol to extend these findings. Third, many of the parents in this study had a psychiatric disorder, which may have introduced a possible source of bias. Their perception of their children's sleep and their motivation to enter their children into the study may not be reflective of the general population. Last, the difference in the age of boys and girls in this study may be a limiting factor with respect to sex differences.

The study's strengths include an assessment of raw EEG power. A previous study ${ }^{13}$ used normalized power to examine the EEG power spectra rather than absolute power values. This method can be vulnerable to insensitivities in group differences. We also measured the child's home environment in order to rule out a chaotic home environment as a possible explanation.

\section{Conclusion}

This study is a first step in evaluating sleep characteristics in boys and girls prior to the diagnosis of a psychiatric disorder. The study suggests that girls, regardless of mood symptoms, have more sleep disturbance than boys their age. Girls with LM show signs of compromised sleep in the first half of the night. Girls with LM, but before clinical diagnosis of depression, show sleep disturbances that may potentially increase their risk for insomnia and psychiatric disorders.

\section{Acknowledgments}

The study was supported by the Michigan Institute for Clinical and Health Research UL1RR024986 to D Conroy and R01 MH77744 to R Armitage. 


\section{Disclosure}

The authors report no conflicts of interest in this work.

\section{References}

1. Singareddy R, Vgontzas A, Fernandez-Mendoza J, et al. Risk factors for incident chronic insomnia: a general population prospective study. Sleep Med. 2012;13:346-353.

2. Breslau N, Roth T, Rosenthal L, Andreski P. Sleep disturbance and psychiatric disorders: a longitudinal epidemiological study of young adults. Biol Psychiatry. 1996;39:411-418.

3. Ford D, Kamerow B. Epidemiologic study of sleep disturbances and psychiatric disorders. An opportunity for prevention? JAMA 1989;262:1479-1484.

4. Weissman MM, Greenwald S, Nino-Murcia G, Dement W. The morbidity of insomnia uncomplicated by psychiatric disorders. Gen Hosp Psychiatry. 1997;19:245-250.

5. Baglioni C, Battagliese G, Feige B, et al. Insomnia as a predictor of depression: a meta-analytic evaluation of longitudinal epidemiological studies. J Affect Disord. 2011;135:10-19.

6. Brower K. Alcohol's effects on sleep in alcoholics. Alcohol Res Health. 2001;25:110-125.

7. Cohn T, Foster J, Peters T. Sequential studies of sleep disturbance and quality of life in abstaining alcoholics. Addict Biol. 2003;8: 455-462.

8. Tsuno N, Besset A, Ritchie K. Sleep and depression. J Clin Psych. 2005;66(10):455-462.

9. Dijk DJ, Brunner D, Beersma D, Borbely AA. Electroencephalogram power density and slow wave sleep as a function of prior waking and circadian phase. Sleep. 1990;13(5):430-440.

10. Brower K, Hoffmann R, Conroy D, Arnedt J, Armitage R. Sleep homeostasis in alcohol-dependent, depressed and healthy control men. Eur Arch Psychiatry Clin Neurosci. 2011;261:559-566.

11. Armitage R, Hoffmann R, Conroy D, Arnedt JT, Brower KJ. Effects of a 3-hour sleep delay on sleep homeostasis in alcohol dependent adults. Sleep. 2012;35(2):273-278.

12. Dahl R, Williamson D, Bertocci M, Stolz M, Ryan N, Ehlers C. Spectral analyses of sleep EEG in depressed offspring of fathers with or without a positive history of alcohol abuse or dependence: a pilot study. Alcohol. 2003;30(3):193-200.

13. Tarokh L, Carskadon M. Sleep electroencephalogram in children with a parental history of alcohol abuse/dependence. J Sleep Res. 2010;19: 165-174.

14. Lopez J, Hoffmann R, Armitage R. Reduced sleep spindle activity in early-onset and elevated risk for depression. J Am Acad Child Adolesc Psychiatry. 2010;49(9):934-943.

15. Robert J, Hoffmann R, Emslie G, et al. Sex and age differences in sleep macroarchitecture in childhood and adolescent depression. Sleep. 2006;29(3):351-358

16. Rao U, Hammen C, Poland R. Risk markers for depression in adolescents: sleep and HPA measures. Neuropsychopharmacology. 2009; 34(8): 1936-1945.

17. Chen C, Burley H, Botlib I. Reduced sleep quality in healthy girls at risk for depression. $J$ Sleep Res. 2012;21:68-72.

18. Colrain I, Baker F. Changes in sleep as a function of adolescent development. Neuropsychol Rev. 2011;21:5-21.

19. Armitage R, Flynn H, Hoffmann R, Vazquez D, Lopez J, Marcus S. Early developmental changes in sleep in infants: the impact of maternal depression. Sleep. 2009;32(5):693-696.

20. Kaufman J, Birmaher B, Brent D. Schedule for Affective Disorders and Schizophrenia for School-Age Children - Present and Lifetime Version (K-SADS-PL). Initial reliability and validity data. $J$ Am Acad Child Adolesc Psychiatry. 1997;36:980-988.

21. Rush J, Trivedi M, Ibrahim H, et al. The 16-item Quick Inventory of Depressive Symptomatology (QIDS), Clinician Rating (QIDS-C), and self report (QIDS-SR): a psychometric evaluation in patients with chronic major depression. Biol Psychiatry. 2003;54(1):573-583.
22. Poznanski E, Freeman L, Mokros H. Children's Depression Rating Scale - Revised (Sep 1984). Psychopharmacol Bull. 1985;21: 979-989.

23. Weinberg W, Emslie G. Weinberg Screening Affective Scales (WSAS and WSAS-SF). J Child Neurol. 1988;3(4):294-296.

24. Shaffer D, Gould M, Brasic J, et al. A children's global assessment scale (CGAS). Arch Gen Psychiatry. 1983;40(11):1228-1231.

25. Moos R, Moos B. Family Environment Scale Manual. Palo Alto, CA: Consulting Psychology Press; 1986.

26. Press W, Flannery B, Teukolosky S, Bettering W, editors. Numerical Recipes in Pascal. New York: Cambridge University Press; 1989.

27. Rechtschaffen A, Kales AA (eds) (1968) A manual of standardized terminology, techniques and scoring system for sleep stages of human subjects. Government Printing Office (NIH Publication No. 204), Washington, DC.

28. Dijk DJ, Beersma D, Daan S. EEG power density during nap sleep: reflection of an hourglass measuring the duration of prior wakefulness. J Biol Rhythms. 1987;2(3):207-219.

29. Dijk DJ, Beersma D, Daan S, Bloem G, Van den Hoofdakker R. Quantitative analysis of the effects of slow wave sleep deprivation during the first $3 \mathrm{~h}$ of sleep on subsequent EEG power density. Eur Arch Psychiatry Neurol Sci. 1987;236(6):323-328.

30. Dijk DJ, Beersma D, Bloem G. Sex differences in the sleep EEG of young adults: visual scoring and spectral analysis. Sleep. 1989;12(6): 500-507.

31. Dijk DJ, Beersma D, Van den Hoofdakker R. All night spectral analysis of EEG sleep in young adult and middle-aged male subjects. Neurobiol Aging. 1989;10(6):677-682.

32. Dijk DJ, Brunner D, Borbely AA. Time course of EEG power density during long sleep in humans. Am J Physiol. 1990;258(3 Pt 2): R650-R661.

33. Dijk DJ. Circadian variation of EEG power spectra in NREM and REM sleep in humans: dissociation from body temperature. $J$ Sleep Res. 1999;8(3):189-195.

34. Feinberg I, March J, Fein G, Floyd T, Walker J, Price L. Period and amplitude analysis of $0.5-3 \mathrm{c} / \mathrm{sec}$ activity in NREM sleep of young adults. Electroencephalogr Clin Neurophysiol. 1978;44(2):202-213.

35. Bertocci M, Dahl R, Williamson D, et al. Subjective sleep complaints in pediatric depression: a controlled study and comparison with EEG measures of sleep and waking. J Am Acad Child Adolesc Psychiatry. 2005;44 (11):1158-1566.

36. Ryan N, Puig-Antich J, Ambrosini P, et al. The clinical picture of major depression in children and adolescents. Arch Gen Psychiatry. 1987;44(10):854-861.

37. Kaneita Y, Ohida T, Osaki Y, et al. Insomnia among Japanese adolescents: a nationwide representative survey. Sleep. 2006;29(12): $1543-1550$.

38. Ohayon M. Epidemiological study on insomnia in the general population. Sleep. 1996;19(Suppl 3):S7-S15.

39. Strine T, Chapman D, Ahluwalia I. Menstrual-related problems and psychological distress among women in the United States. $J$ Womens Health (Larchmt). 2005;14(4):316-323.

40. Fulton MK, Armitage R, Rush AJ. Sleep electroencephalographic coherence abnormalities in individuals at high risk for depression: A pilot study. Biol Psychiatry. 2000;47:618-625.

41. Perlis ML, Merica H, Smith MT, Giles D. Beta EEG activity and insomnia. Sleep Med Rev. 2001;5(5):365-376.

42. Armitage R, Hoffmann R, Fitch T, Trivedi M, Rush A. Temporal characteristics of delta activity during NREM sleep in depressed outpatients and healthy adults: group and sex effects. Sleep. 2000;23(5):607-617.

43. Tarokh L, Carskadon M, Achermann P. Dissipation of sleep pressure is stable across adolescence. Neuroscience. 2012;216:167-177.

44. Emslie G, Kennard B, Mayes T, et al. Insomnia moderates outcome of serotonin-selective reuptake inhibitor treatment in depressed youth. J Child Adolesc Psychopharmacol. 2012;22(1):21-28.

45. Shamseddeen W, Clarke G, Keller M, et al. Adjunctive sleep medications and depression outcome in the treatment of serotonin-selective reuptake inhibitor resistant depression in adolescents study. $J$ Child Adolesc Psychopharmacol. 2012;22(1):29-36. 


\section{Publish your work in this journal}

Nature and Science of Sleep is an international, peer-reviewed, open access journal covering all aspects of sleep science and sleep medicine, including the neurophysiology and functions of sleep, the genetics of sleep, sleep and society, biological rhythms, dreaming, sleep disorders and therapy, and strategies to optimize healthy sleep. The journal welcomes

original research, clinical \& epidemiological studies, reviews \& evaluations, case reports and extended reports. The manuscript management system is completely online and includes a very quick and fair peerreview system, which is all easy to use. Visit http://www.dovepress.com/ testimonials.php to read real quotes from published authors.

Submit your manuscript here: http://www.dovepress.com/nature-and-science-of-sleep-journal 